\title{
sciendo
}

\section{Similar Muscular Adaptations in Resistance Training Performed Two Versus Three Days Per Week}

\author{
by \\ Thiago Lasevicius', Brad Jon Schoenfeld², Jozo Grgic ${ }^{3}$, Gilberto Laurentino', \\ Lucas Duarte Tavares ${ }^{1}$, Valmor Tricoli ${ }^{1}$
}

The purpose of the present study was to compare changes in muscle strength and hypertrophy between volumeequated resistance training (RT) performed 2 versus 3 times per week in trained men. Thirty-six resistance-trained men were randomly assigned to one of the two experimental groups: a split-body training routine (SPLIT) with muscle groups trained twice per week $(n=18)$ over four weekly sessions, or a total-body routine (TOTAL), with muscle groups being trained three times per week $(n=18)$ over three weekly sessions. The training intervention lasted 10 weeks. Testing was carried out pre- and post-study to assess maximal muscular strength in the back squat and bench press, and hypertrophic adaptations were assessed by measuring muscle thickness of the elbow flexors, elbow extensors, and quadriceps femoris. Twenty-eight subjects completed the study. Significant pre-to-post intervention increases in upper and lower-body muscular strength occurred in both groups with no significant between-group differences. Furthermore, significant preto-post intervention increases in muscle size of the elbow extensors and quadriceps femoris occurred in both groups with no significant between-group differences. No significant pre-to-post changes were observed for the muscle size of elbow flexors both in the SPLIT or TOTAL group. In conclusion, a training frequency of 2 versus 3 days per week produces similar increases in muscular adaptations in trained men over a 10-week training period. Nonetheless, effect size differences favored SPLIT for all hypertrophy measures, indicating a potential benefit for training two versus three days a week when the goal is to maximize gains in muscle mass.

Key words: frequency, strength training, hypertrophy, volume.

\section{Introduction}

The manipulation of resistance training (RT) variables is thought to be paramount for maximizing muscular adaptations in humans (Kraemer and Ratamess, 2004). The American College of Sports Medicine (2009) recommends that resistance trained individuals should perform a majority of repetitions with a load corresponding to 6-12 repetition maximum (RM), using a movement tempo of 1-2 seconds per muscle action (i.e. concentric and eccentric phase), and taking 1 to 2 minutes rest between sets. Furthermore, individuals should perform at least 10 sets per week per muscle group to maximize muscular adaptations (Schoenfeld et al., 2017). However, research is somewhat limited as to the effects of manipulating RT frequency. RT frequency can be defined as the number of weekly RT sessions. Frequency can also be characterized as how many times a given muscle group is worked each week a definition that is more relevant to bodybuilders and others seeking to maximize muscle growth (Hackett et al., 2013).

The acute post-exercise muscle protein synthesis (MPS) response to RT lasts $\sim 48$ hours in untrained individuals (Phillips et al., 1997) and there is evidence that this response is attenuated in those acclimated to RT, whereby the time course is

\footnotetext{
1 - Department of Sport, School of Physical Education and Sport, University of São Paulo, São Paulo, SP, Brazil.

2 - Health Sciences Department, CUNY Lehman College, Bronx, NY, USA.

3 - Institute for Health and Sport (IHES)Victoria University, Melbourne, Australia.
} 
reduced to $\sim 36$ hours or less (MacDougall et al., 1995; Tang et al., 2008). Based on this attenuated MPS response in trained individuals, some have speculated that an increased RT frequency would sustain elevations in MPS over time, thereby maximizing the area under the curve and, in theory, result in a greater muscle protein accretion (Dankel et al., 2017). Consistent with motor learning theory, it also can be surmised that performing a given exercise more frequently over time would elicit superior increases in strength, conceivably through heightened improvements in neural efficiency (Shea et al., 2000). However, the hypothesis that greater RT frequencies enhance muscular adaptations remains speculative. Such inferences can only be gleaned from longitudinal studies that directly compare different RT frequencies while controlling all other variables.

A recent meta-analysis of longitudinal studies on the topic of RT frequency found that training a muscle group twice per week produced superior gains in muscle mass as compared to a RT frequency of once per week (Schoenfeld et al., 2016). However, there were insufficient data to determine whether even higher frequencies of training would further enhance the hypertrophic response. In addition, only two out of 10 studies included in the meta-analysis involved resistancetrained individuals, and both studies compared training muscle groups once versus three times per week (McLester et al., 2000; Schoenfeld et al., 2015). Moreover, all of the studies that investigated training frequencies of two versus three days per week employed whole body measures of muscle mass (e.g., dual-energy X-ray absorptiometry), which are not as sensitive for detecting subtle changes over time as site-specific measures such as ultrasound or magnetic resonance imaging (Levine et al., 2000; Maden-Wilkinson et al., 2013). It is evident that there is a paucity of research investigating the potential benefits of training muscle groups at frequencies of two or more days per week while using site-specific measures of muscle growth and performed in those with previous RT experience. Therefore, in an effort to fill important gaps in the current literature, the purpose of this paper was to compare changes in muscular strength and hypertrophy between RT performed two versus three days per week in a group of resistance-trained men. In order to control for potential confounding factors, the total weekly
RT volume (sets $x$ repetitions $x$ load) was equated between groups as research shows that there is a direct dose-response relationship between volume and gains in both muscular strength and muscle mass (Ralston et al., 2017; Schoenfeld et al., 2017). Our hypothesis was that training muscle groups three times per week would promote greater muscular adaptations compared to a weekly training frequency of twice per week.

\section{Methods}

\section{Participants}

The participants were 36 apparently healthy male volunteers (age $=21.0 \pm 3.0$ yrs; body mass $=$ $78.7 \pm 9.8 \mathrm{~kg}$; body height $=178.5 \pm 6.0 \mathrm{~cm}$; RT experience $=3.2 \pm 1.1 \mathrm{yrs}$ ); the sample size was justified by a power analysis with the outcome being vastus lateralis muscle thickness (MT) with an effect size (ES) difference of $0.40, p=.05$ and power of 0.80 while factoring in the possibility of six dropouts. Participants were considered to be resistance-trained, defined as having a minimum consistent RT experience of at least three days-perweek (on most weeks) for one year. All participants employed the bench press and squat exercises in their usual training routines. Based on the baseline strength and MT, the participants were pairmatched and then randomly assigned to a splitbody routine (SPLIT) whereby muscle groups were trained twice per week $(n=18)$ over four weekly sessions, or a total-body routine (TOTAL), where muscle groups were trained three times per week $(n=18)$ over three weekly sessions. The University of São Paulo Institutional Review Board approved the research protocol, and all participants signed an informed consent form.

\section{Resistance training procedures}

During the 10-week training period, the participants were instructed not to do any additional (i.e., external) RT. The specific protocols for SPLIT and TOTAL routines are outlined in Table 1 . The number of repetitions per set was 812 , with sets carried out to the point of momentary concentric muscular failure. The rest interval between sets was $90 \mathrm{~s}$. The cadence of repetitions for concentric and eccentric actions was 2:2. All training sessions were supervised by research personnel experienced in RT performance. Before the training program started, the participants were tested for their 10 repetition maximum (RM) in all of the exercises employed during the intervention. The 10RM data were used to determine 
participants' initial training loads. During the course of the study, we attempted to progressively increase the external load by $2.5 \%$ when participants performed more than 12 repetitions for a given set of an exercise; alternatively, loads were decreased by $2.5 \%$ when they performed fewer than 8 repetitions for a given set of an exercise.

\section{Dietary adherence}

The participants were advised to maintain their usual and customary nutritional habits and to avoid consuming any muscle-building supplements during the study period. The MyFitnessPal.com (http://www.myfitnesspal.com) software was used for the collection of food records. The food records were self-reported by the participants twice: the first time was seven days before the training program started and the second time was in week ten of the intervention. The participants were instructed by the research staff on how to use the software and how to report their food items. Total values of protein, carbohydrates, and fats were recorded for further analysis.

\section{Muscle thickness}

B-mode ultrasound was used for the assessment of MT (imaging unit: SonoAce R3, Samsung-Medison, Gangwon-do, South Korea). The assessment of MT was performed by an experienced technician. Following the application of water-soluble transmission gel (Aquasonic 100 Ultrasound Transmission gel, Parker Laboratories Inc., Fairfield, NJ), the technician used a five $\mathrm{MHz}$ ultrasound probe, which was placed perpendicularly to the tissue interface with caution been taken not to depress the skin. Upon obtaining a satisfactory quality image, the image was saved to hard drive. MT was measured according to the protocol utilized by Abe et al. (2000). Measurements were taken at two sites for the upper-body (elbow flexors and elbow extensors) and at two sites for the lower-body (rectus femoris and vastus lateralis). For the upper-body, assessment of MT was done at a site located $60 \%$ distal between the humerus lateral epicondyle and the acromion process of the scapula. For the lowerbody, the assessment was done at a site located $50 \%$ between the greater trochanter and the lateral condyle of the femur. In order to reduce the confounding impact of training-induced edema, the assessment of MT was carried out 48-72 hours after the final training session (Ogasawara et al.,
2012). The typical error (TE) and the coefficient of variation for MT measurements of the elbow flexor, elbow extensor, rectus femoris and vastus lateralis were $0.8 \mathrm{~mm}(\mathrm{CV}: 2.17 \%), 0.6 \mathrm{~mm}(\mathrm{CV}$ : $2.13 \%), 1.0 \mathrm{~mm}(\mathrm{CV}: 3.85 \%)$, and $1.0 \mathrm{~mm}(\mathrm{CV}$ : $3.95 \%)$, respectively.

\section{Muscle strength}

Lower-body muscular strength was evaluated using the 1RM Smith squat test (1RMsQuat), while upper-body muscular strength was assessed using the 1RM barbell bench press test (1RMBENCH). For testing at baseline and post-RT intervention, the participants reported to the lab having refrained from any exercise for at least 48 hours. Upon arriving at the lab, the participants performed a warm-up which consisted of light cardiovascular exercise ( 10 $\mathrm{min})$. The first set of a given exercise was carried out at a load of $\sim 50 \%$ of the 1RM for five repetitions. The second and third sets were performed with two-three repetitions at a load corresponding to $\sim 60-80 \%$ of the $1 \mathrm{RM}$. The following sets were carried out with one repetition while increasing weight for the 1RM determination and resting from 3 to 5 minutes between each successive attempt. All 1RM determinations were made within 5 attempts. The participants' thighs were required to reach parallel in the 1RMsQuAT for the attempt to be considered successful as determined by the research assistants. Successful 1 RMBENCH was achieved if the participant displayed a 5-point body contact position (head, upper back, and buttocks firmly on the bench with both feet flat on the floor) and executed a full lock out of the limbs. 1RMBench testing was conducted first in the sequence; the 1RMsquat test was carried out following a 5-min rest period. Hand and foot placement during the muscular strength tests were recorded at baseline, and the same positions were reused for post-study performance.

The 10RM test was performed in the following order: Smith squat, bench press, leg press $45^{\circ}$, lat pulldown, leg extension, triceps pushdown and biceps curl. The exercises were selected because of their extensive use in RT programs and ease of execution. Standardized instructions were provided to participants prior to the test. Final values were obtained within 3 attempts, with 3- to 5-min rest intervals afforded between attempts. After obtaining the load in a given exercise, recovery intervals of no less than 5 min were provided before proceeding to test $10 \mathrm{RM}$ 
for the ensuing exercise.

10RM testing for the bench press and Smith squat exercises followed the same procedures as employed for the 1RM test. Procedures for the other exercises were as follows:

1) Leg press $45^{\circ}$ : the footplate of the unit was divided into $10 \times 10 \mathrm{~cm}$ squares with adhesive tape to facilitate annotation of the feet positioning on a sheet of paper and thus ensure reproducibility in all testing sessions. Thereafter, the range of motion for each repetition was determined by having participants perform an unloaded repetition, starting with the complete extension of the knees up to $90^{\circ}$ of flexion; a goniometer was used to confirm the degree of flexion. A plastic marker was then placed on the side column of the leg press $45^{\circ}$ to mark the knee flexion point. A measuring tape was also glued onto the side column of the apparatus to ensure reproducibility of marker positioning.

2) Lat pulldown: participants sat on the bench of the lat pulldown machine with the trunk slightly inclined and knees semi-flexed. They grasped the bar using a shoulder-width grip in full elbow extension, and then pulled the bar down to the chest by simultaneously adducting at the shoulder joint and flexing at the elbow.

3) Leg extension: participants sat upright with the trunk fully supported on the backrest of extensor chair and knees at a $90^{\circ}$ angle. Movement was initiated by extending the knees until reaching full extension and then flexing them back to the initial position.

4) Triceps pushdown: participants assumed a shoulder-width stance, knees semiflexed, elbows flexed at a $90^{\circ}$ angle, and hands holding the bar in a pronated position. Movement entailed pushing down on the bar until achieving complete elbow extension.

5) Biceps curl: participants assumed a shoulder-width stance, knees semi-flexed, elbows extended and hands holding the bar in a supinated position. Movement entailed curling the bar until achieving complete elbow flexion.

After obtaining the maximum loads in the 10RM test, participants rested for 48 hours and were reevaluated to obtain the reproducibility of the test (test and retest). The load established on both days, a difference of less than $5 \%$ was considered as the 10RM. In the intervals between testing sessions, participants were instructed not to perform any type of resistive exercise. The initial loads used in the experimental conditions were the highest obtained during pre-testing. The intraclass correlation coefficients (ICC) for the tests were as follows: Smith squat: ICC $=0.98$; bench press: ICC $=0.99$; leg press 45․ ICC $=1.00$; lat pulldown: ICC = 0.99; leg extension: $\mathrm{ICC}=0.99$; triceps pushdown: ICC $=0.97$; biceps curl ICC $=0.99$.

\section{Statistical analysis}

Initially, the data were analyzed quantitatively and visually to verify their normality (Shapiro-Wilk test) and existence of outliers (box-plots). The TE of measurement was used for measures of reliability, calculated as the standard deviation of the difference between day one and day two measurements / $\sqrt{2}$ and the coefficient of variation calculated as the (TE of the difference between day one and day two / means of the day one and day two values) ${ }^{*} 100$. A oneway ANOVA was performed to compare differences between groups in the volume load and dietary intake. A mixed model analysis was performed for each dependent variable (MT and 1RM), with groups (TOTAL and SPLIT) and time (pre- and post-intervention) as fixed factors, and participants as a random factor. For each outcome, an ES was calculated as proposed by Morris (2008). The ES magnitude was classified as follows: $<0.20$ was considered as trivial, 0.20-0.50 was considered as small, $0.50-0.80$ as moderate and $>0.80$ was considered as being of large magnitude (Cohen, 1988). Pre- to post RT intervention percent changes were also calculated for the muscular strength and hypertrophy outcomes ([post-testing value - pretesting value] / pre-testing value * 100). The statistical significance threshold was set at $p<.05$. All analyses were performed using the statistical software package SAS 9.2 (SAS, NC).

\section{Results}

Eight participants dropped out over the course of the study (four from each group). The participants dropped out due to personal reasons (non-related to the RT intervention). Thus, a total of 28 participants completed the study (14 in each group). Attendance was $95.6 \%$ and $95.3 \%$ for the TOTAL and SPLIT group, respectively.

\section{Dietary analyses}

There were no significant differences in average caloric intake or consumption of protein, carbohydrate or fat between the TOTAL and SPLIT groups. Data of daily dietary intake are presented 
in Table 2.

\section{Volume load}

Volume load was calculated as sets $x$ repetitions $x$ load for all sets performed during the entire study. There were no differences in the volume load between groups for chest $(p=.89 ; 95 \%$ confidence interval $[\mathrm{CI}]=-20912.0,18363.5)$, back $(p=.79 ; 95 \% \mathrm{CI}=-13364.7,17270.9)$, elbow flexors $(p=.25 ; 95 \% \mathrm{CI}=-2600.9,9489.2)$, elbow extensors $(p=.74 ; 95 \% \mathrm{CI}=-24719.9,17869.2)$ and anterior thigh $(p=.78 ; 95 \% \mathrm{CI}=-74560.3,100729.0)$. Results are presented in Table 3.

\section{Muscle thickness}

MT of the rectus femoris increased from baseline to post-study by $7.9 \%(p<.001)$ and $12.3 \%$ $(p<.001)$ for TOTAL and SPLIT groups, respectively, with no statistical differences noted between groups $(p=.50 ; \mathrm{CI}=-0.19,0.09$; ES difference $=0.39)($ Table 4$)$.

MT of the vastus lateralis increased from baseline to post-study by $12.2 \%(p<.001)$ and $16.9 \%$ $(p<.001)$ for TOTAL and SPLIT groups, respectively, with no statistical differences noted between groups $(p=.54 ; 95 \% \mathrm{CI}=-0.25,0.13$; ES difference $=0.31)($ Table 4$)$.

There were no statistical differences noted for time or between groups in MT of the elbow flexors $(p=.67 ; 95 \% \mathrm{CI}=-0.22,0.34 ; \mathrm{ES}$ difference $=0.36)$ (Table 4). The change from baseline to post-study was $1.6 \%(p>.05)$ and $7.3 \%(p>.05)$ for TOTAL and SPLIT groups, respectively.

MT of the elbow extensors increased from baseline to post-study by $8.6 \%(p<.001)$ and $15.7 \%$ $(p<.001)$ for TOTAL and SPLIT groups, respectively, with no statistical differences noted between groups $(p=.62 ; 95 \% \mathrm{CI}=-0.45,0.27$; ES difference $=0.35)($ Table 4$)$.

\section{Muscle strength}

There was a significant main effect of time for the 1 RMBench $(p=.033)$ and 1RMsquat $(p=.0001)$. 1RMвеNCH increased from baseline to post-study by $10.2 \%(p=.034)$ and $11.8 \%(p=.03)$ for TOTAL and SPLIT groups, respectively, with no differences noted between groups $(p=.47 ; 95 \% \mathrm{CI}=-13.75$, 6.54; ES difference $=0.09)$ (Table 4). 1RMsQuat increased from baseline to post-study by $17.7 \%$ ( $p$ $=.011)$ and $18.9 \%(p=.006)$ for TOTAL and SPLIT groups, respectively, with no differences noted between groups ( $p=.60 ; 95 \% \mathrm{CI}=-21.54,12.61$; ES difference $=0.09)($ Table 4$)$.

\section{Discussion}

The present study is the first to investigate the effects of training muscle groups two versus three days per week in men with previous RT experience while equating total training volume between conditions using site-specific measures of muscle growth. The primary and novel finding of our study is that both RT frequencies elicited similar increases in muscular strength and hypertrophy over a 10-week program. The results are not in agreement with our hypothesis that the TOTAL group would experience greater increases in muscle size and strength, and appear to refute the hypothesis that increasing the frequency of MPS stimulation with RT necessarily leads to enhanced muscle protein accretion over time.

Both TOTAL and SPLIT produced significant gains in maximal strength with marked increases noted in the 1 RMBENCH $(10.2 \%$ and $11.8 \%$, respectively) and 1RMsquat $(17.7 \%$ and $18.9 \%$, respectively). No statistical differences were found between conditions and the ES differences were minimal for both strength outcomes. These results seem to be in line with several recent studies that compared RT frequency prescription and were done in resistance-trained individuals. For example, Yue et al. (2018) compared training a muscle group two versus four times per week with volume equated between conditions and observed no significant differences in strength gains between the two groups. Brigatto et al. (2018) compared strength gains over an 8-week period between groups training a muscle group once versus twice per week on a volume-equated basis. Both groups increased their levels of strength in the bench press and squat exercise post-intervention; however, no significant differences between the training groups were found. Furthermore, our results corroborate meta-analytical data which reported that when training volume was equated, no significant effect of RT frequency on strength gains was observed (Grgic et al., 2018). 


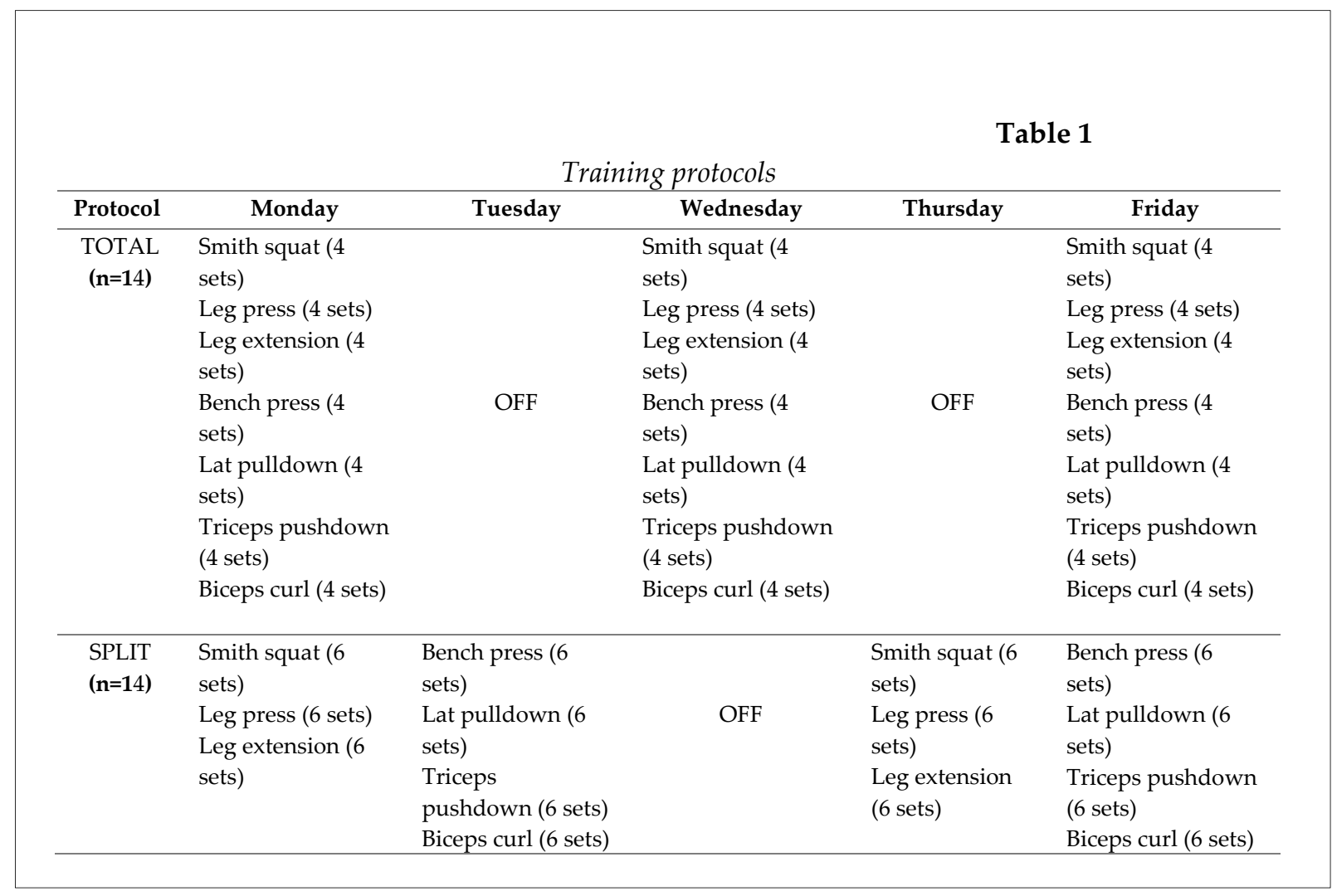

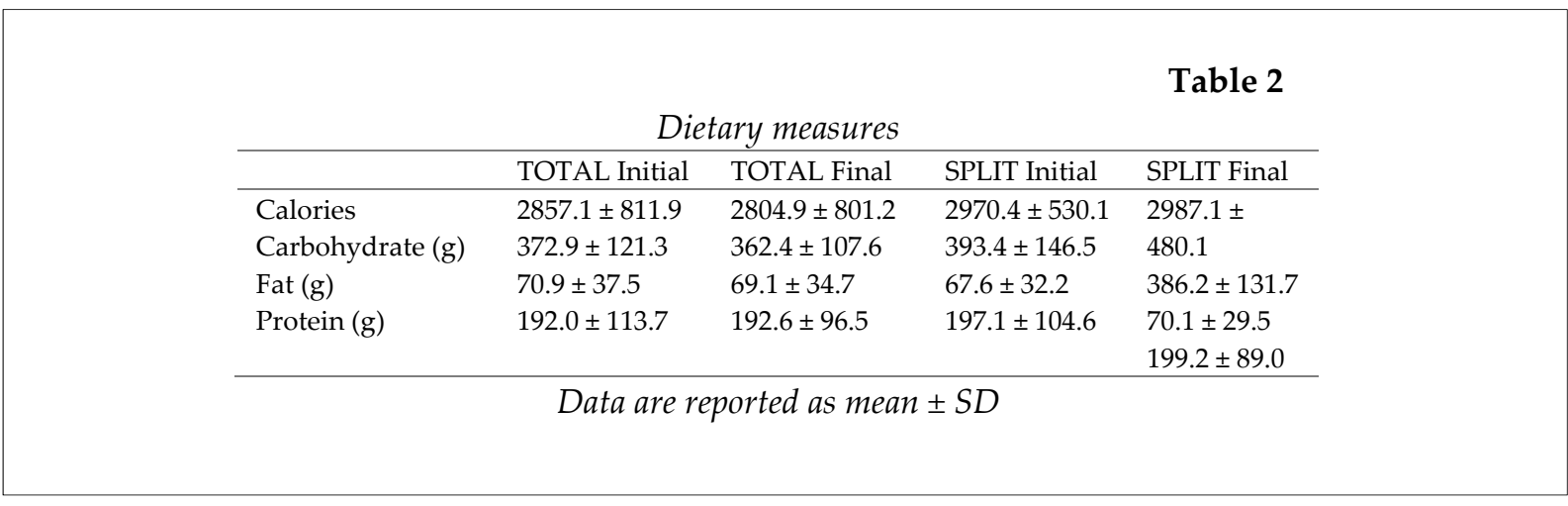

\begin{tabular}{|c|c|c|}
\hline \multicolumn{3}{|c|}{ Total volume load by exercise $(\mathrm{kg})$} \\
\hline $\begin{array}{l}\text { Muscle Group } \\
\text { Bench press } \\
\text { Lat pulldown } \\
\text { Biceps curl } \\
\text { Triceps pushdown } \\
\text { Smith Squat } \\
\text { Leg Press } 45^{\circ} \\
\text { Leg Extension } \\
\end{array}$ & $\begin{array}{c}\text { TOTAL } \\
62154.6 \pm 27848.0 \\
63444.5 \pm 21597.2 \\
34527.7 \pm 7749.4 \\
59001.4 \pm 25613.2 \\
185532.7 \pm 92670.7 \\
244459.2 \pm 61992.5 \\
81486.4 \pm 20664.1 \\
\end{array}$ & $\begin{array}{c}\text { SPLIT } \\
63428.9 \pm 27848.0 \\
61491.4 \pm 17635.5 \\
31083.6 \pm 7812.0 \\
62426.8 \pm 29094.2 \\
158611.2 \pm 33466.6 \\
237917.1 \pm 50199.9 \\
79305.5 \pm 16733.3 \\
\end{array}$ \\
\hline \multicolumn{3}{|c|}{ Data are reported as mean $\pm S D$. } \\
\hline
\end{tabular}


Table 4

Pre- to Post-study outcome measures

\begin{tabular}{llllllc}
\hline Measure & \multicolumn{1}{c}{ Total-Pre } & \multicolumn{1}{c}{ Total-Post } & ES & \multicolumn{1}{c}{ Split-Pre } & \multicolumn{1}{c}{ Split-Post } & ES \\
Rectus Femoris (mm) & $22.7 \pm 2.5$ & $24.5 \pm 2.7^{*}$ & 0.71 & $22.7 \pm 2.6$ & $25.5 \pm 2.2^{*}$ & 1.10 \\
Vastus Lateralis (mm) & $21.2 \pm 3.4$ & $23.8 \pm 3.9^{*}$ & 0.83 & $21.3 \pm 2.9$ & $24.9 \pm 3.1^{*}$ & 1.14 \\
Elbow Flexors (mm) & $35.9 \pm 5.3$ & $36.5 \pm 6.0$ & 0.11 & $34.3 \pm 5.3$ & $36.8 \pm 4.7$ & 0.47 \\
Elbow Extensor (mm) & $30.0 \pm 5.6$ & $32.6 \pm 8.1^{*}$ & 0.43 & $29.9 \pm 6.5$ & $34.6 \pm 5.8^{*}$ & 0.78 \\
1RM Squat $(\mathrm{kg})$ & $156.5 \pm 26.5$ & $184.3 \pm 31.2^{*}$ & 1.03 & $159.7 \pm 27.7$ & $190.0 \pm 29.3^{*}$ & 1.12 \\
1RM Bench Press (kg) & $78.1 \pm 19.8$ & $86.1 \pm 21.6^{*}$ & 0.45 & $80.9 \pm 15.6$ & $90.5 \pm 17.3^{*}$ & 0.54 \\
\hline
\end{tabular}

Data are reported as mean $\pm S D$. Asterisk ${ }^{*}$ ) indicates a significant effect from baseline

values. $E S=$ effect size

These findings are somewhat counterintuitive, as motor learning theory dictates that practicing a given exercise more frequently over time leads to better skill acquisition, conceivably through neural enhancements (Shea et al., 2000). Given our results, it may be speculated that training twice a week is sufficient to optimize neural proficiency in strength-related tasks, and thereafter, training volume (and not frequency) becomes the predominant factor for maximizing results. Alternatively, it is possible that there is not a sufficient difference between two versus three weekly RT sessions from a frequency standpoint, and perhaps higher frequencies are required to promote additional neural improvements. That said, this idea remains speculative given that Colquhoun et al. (2018) and Saric et al. (2018) recently compared volume-equated training three versus six times per week and observed comparable strength gains between the two training frequencies. Further research is required to develop a better understanding of these complexities.

With respect to gains in muscle mass, while both groups increased MT of the elbow extensors and quadriceps following 10 weeks of training, no significant between-group differences were seen in the upper- or lower-body. No previous studies have endeavored to evaluate sitespecific changes in muscle size when training muscle groups two versus three days per week. However, several volume-equated investigations of training frequency have employed whole-body measures of muscle mass including girth measurements (Arazi and Asadi, 2011), air displacement plethysmography (Benton et al., 2011), and dual x-ray absorptiometry (Candow and Burke, 2007), with no significant betweengroup differences reported in all of these studies. Schoenfeld et al. (2015) found greater increases in elbow flexor MT and a trend for greater increases in vastus lateralis MT when comparing volumeequated groups training muscle groups three times versus once per week. Brigatto et al. (2018) compared training once versus twice per week (over an 8-week training period) and reported no significant differences in muscle hypertrophy between the training groups. Based on the current body of evidence, it is possible that variables such as training volume may have a more profound effect on muscular hypertrophy as compared to RT frequency (Schoenfeld et al., 2017; Schoenfeld et al., 2019). It should be noted that small, but potentially meaningful ES differences (ranging from 0.31 to 0.39 ) were observed in favor of training muscles two versus three days per week for every hypertrophy outcome measure studied. These findings suggest a potential hypertrophic benefit to the lower training frequency. That said, additional studies using direct measures of 
hypertrophy (i.e., ultrasound, magnetic resonance imaging or computed tomography) are warranted to explore this topic further. Also, studies with longer duration time courses are needed given that the majority of the current studies were six to 10 weeks in duration.

One possible limitation of the present study is that it might have been slightly underpowered from a statistical standpoint. We initially recruited a sample of 36 participants; however, eight participants did not complete the whole training program, which subsequently might have impacted the statistical power of the study. Additionally, the sample was comprised of young. trained men which therefore limits the generalizability of the results to those who are untrained, older, and women. Finally, the training program lasted 10 weeks; it is possible that the results would be different over longer time courses.

\section{Conclusions}

Based on our findings, we conclude that training muscle groups either twice or three times per week results in similar increases in muscular strength and hypertrophy in young adult trained men when volume is equated between the training conditions. The TOTAL protocol employed in this study required fewer weekly sessions, but more training time per session compared to SPLIT. Alternatively, the SPLIT protocol required a higher total number of weekly sessions, but each session was of shorter duration compared to TOTAL. Given the larger ESs favoring the SPLIT training routine noted for hypertrophic outcomes, it remains possible that small, but potentially meaningful improvements may be elicited by employing a training frequency of twice per week as compared to training three times per week.

\section{References}

Abe T, DeHoyos DV, Pollock ML, Garzarella L. Time course for strength and muscle thickness changes following upper and lower body resistance training in men and women. Eur J Appl Physiol, 2000; 81: 174180

American College of Sports Medicine. American College of Sports Medicine position stand. Progression models in resistance training for healthy adults. Med Sci Sports Exerc, 2009; 41(3): 687

Arazi H, Asadi A. Effects of 8 weeks equal-volume resistance training with different workout frequency on maximal strength, endurance and body composition. Int J Sports Sci Eng, 2011; 5: 11-8

Baechle TR, Earle RW. (Eds.). Essentials of strength training and conditioning (3rd ed.). Champaign, IL: Human Kinetics; 2008

Benton MJ, Kasper MJ, Raab SA, Waggener GT, Swan PD. Short-term effects of resistance training frequency on body composition and strength in middle-aged women. J Strength Cond Res, 2011; 25: 3142-9

Brigatto FA, Braz TV, Zanini TCDC, Germano MD, Aoki MS, Schoenfeld BJ, Marchetti PH, Lopes CR. Effect of resistance training frequency on neuromuscular performance and muscle morphology after eight weeks in trained men. J Strength Cond Res, 2018. doi: 10.1519/JSC.0000000000002563

Candow DG, Burke DG. Effect of short-term equal-volume resistance training with different workout frequency on muscle mass and strength in untrained men and women. J Strength Cond Res, 2007; 21: 204207

Cohen J. Statistical power analysis for the behavioral sciences, 2nd edition. Hillsdale; NJ: Lawrence Erlbaum; 1998

Colquhoun RJ, Gai CM, Aguilar D, Bove D, Dolan J, Vargas A, Couvillion K, Jenkins NDM, Campbell BI. Training volume, not frequency, indicative of maximal strength adaptations to resistance training. $J$ Strength Cond Res, 2018; 32: 1207-1213

Dankel SJ, Mattocks KT, Jessee MB, Buckner SL, Mouser JG, Counts BR, Laurentino GC, Loenneke JP. Frequency: The overlooked resistance training variable for inducing muscle hypertrophy? Sports Med, 2017; 47: 799-805

Grgic J, Schoenfeld, BJ, Davies TB, Lazinica B, Krieger JW, Pedisic Z. Effect of resistance training frequency on gains in muscular strength: a systematic review and meta-analysis. Sports Med, 2018; 48: 1207-1220 
Hackett DA, Johnson NA, Chow CM. Training practices and ergogenic aids used by male bodybuilders. J Strength Cond Res, 2013; 27: 1609-1617

Kraemer WJ, Ratamess NA. Fundamentals of resistance training: Progression and exercise prescription. Med Sci Sports Exerc, 2004; 36: 674-688

Levine JA, Abboud L, Barry M, Reed JE, Sheedy PF, Jensen MD. Measuring leg muscle and fat mass in humans: Comparison of CT and dual-energy X-ray absorptiometry. J Appl Physiol (1985), 2000; 88: 452-456

MacDougall JD, Gibala MJ, Tarnopolsky MA, MacDonald JR, Interisano SA, Yarasheski KE. The time course for elevated muscle protein synthesis following heavy resistance exercise. Can J Appl Physiol, 1995; 20: $480-486$

Maden-Wilkinson TM, Degens H, Jones DA, McPhee JS. Comparison of MRI and DXA to measure muscle size and age-related atrophy in thigh muscles. J Musculoskelet Neuronal Interact, 2013; 13: 320-8

McLester JH, Bishop E, Guilliams ME. Comparison of 1 day and 3 days per week of equal-volume resistance training in experienced subjects. J Strength Cond Res, 2000; 14: 273-281

Morris B. Estimating effect sizes from pretest-posttest-control group designs. Org Res Met, 2008; 11: 364-386

Ogasawara R, Thiebaud RS, Loenneke JP, Loftin M, Abe T. Time course for arm and chest muscle thickness changes following bench press training. Interv Med Appl Sci, 2012; 4: 217-220

Phillips SM, Tipton KD, Aarsland A, Wolf SE, Wolfe RR. Mixed muscle protein synthesis and breakdown after resistance exercise in humans. Am J Physiol, 1997; 273: E99-107

Ralston GW, Kilgore L, Wyatt FB, Baker JS. The effect of weekly set volume on strength gain: A meta-analysis. Sports Med, 2017; 47: 2585-2601

Saric J, Lisica D, Orlic I, Grgic J, Krieger JW, Vuk S, Schoenfeld BJ. Resistance Training Frequencies of 3 and 6 Times Per Week Produce Similar Muscular Adaptations in Resistance-Trained Men. J Strength Cond Res, 2018. doi: 10.1519/JSC.0000000000002909

Schoenfeld BJ, Grgic J, Krieger J. How many times per week should a muscle be trained to maximize muscle hypertrophy? A systematic review and meta-analysis of studies examining the effects of resistance training frequency. J Sports Sci, 2019; 37: 1286-1295

Schoenfeld BJ, Ogborn D, Krieger JW. Effects of resistance training frequency on measures of muscle hypertrophy: A systematic review and meta-analysis. Sports Med, 2016; 46: 1689-1697

Schoenfeld BJ, Ogborn D, Krieger JW. Dose-response relationship between weekly resistance training volume and increases in muscle mass: A systematic review and meta-analysis. J Sports Sci, 2017; 35: 1073-1082

Schoenfeld BJ, Ratamess NA, Peterson MD, Contreras B, Tiryaki-Sonmez G. Influence of resistance training frequency on muscular adaptations in well-trained men. J Strength Cond Res, 2015; 29(7): 1821-1829

Shea $\mathrm{CH}$, Lai Q, Black C, Park JH. Spacing practice sessions across days benefits the learning of motor skills. Hum Mov Sci, 2000; 19: 737-760

Tang JE, Perco JG, Moore DR, Wilkinson SB, Phillips SM. Resistance training alters the response of fed state mixed muscle protein synthesis in young men. Am J Physiol Regul Integr Comp Physiol, 2008; 294: R172-8

Yue FL, Karsten B, Larumbe-Zabala E, Seijo M, Naclerio F. Comparison of 2 weekly-equalized volume resistance-training routines using different frequencies on body composition and performance in trained males. Appl Physiol Nutr Metab, 2018; 43: 475-481

\section{Corresponding author:}

\section{Brad Jon Schoenfeld}

Health Sciences Department, CUNY Lehman College,

Bronx, NY, 250 Bedford Park Blvd West, Bronx, NY 10468,

Phone: 718-960-1999;

E-mail: bradschoenfeldphd@gmail.com 